\title{
Unique Analysis Approach to Bridge-T Network using Floating Admittance Matrix Method
}

\author{
Sanjay Kumar Roy \\ $\mathrm{Ph} . \mathrm{D}$. Scholar Lovely \\ Professional University and Head \\ (Power Distribution), OMQ \\ Division, TATA Steel Ltd \\ India \\ sanjay.roy@tatasteel.com
}

\author{
Kamal Kumar Sharma \\ Professor, Electronics \& \\ Communication Engineering, \\ Lovely Professional University, \\ Phagwara, Punjab, India \\ kamalsharma111@gmail.com
Brahmadeo Prasad Singh
Professor Adjunct,
Technology,
New Delhi, India
bpsinghgkp@gmail.com \\ Netaji Subhas University of
}

\author{
Cherry Bhargava \\ Associate Professor, Computer \\ Science and Engineering, \\ Symbiosis Institute of \\ Technology, Pune, India \\ cherry.bhargava@sitpune.edu.in
}

Received: February 12, 2021. Revised: August 19, 2021. Accepted: September 3, 2021. Published: September 6, 2021.

\begin{abstract}
The RC bridge-T Circuit are sometimes preferred for radio frequency applications as it does not require transformer (inductive coupling). The uses of the resistance-capacitance form of the network permits a wide tuning range. The article aims to develop a band pass filter's mathematical model using the Floating Admittance Matrix (FAM) approach.
\end{abstract}

Both types of RC bridge-T network form the bandpass filters. The use of the conventional methods of analysis such as KCL, KVL, Thevenin's, Norton's depends on its suitability for the type of the particular circuit.

The proposed mathematical modeling scheme using the floating admittance matrix approach is unique, and the same can be used for all types of circuits. This method is suitable to use the partitioning technique for large network. The sum property of all the elements of any row or any column equal to zero provides the assurance to proceed further for analysis or re-observe the very first equation. This saves time and energy. The FAM method presented here is so simple that anybody with slight knowledge of electronics but understating the matrix maneuvering, can analyze any circuit to derive all types of its transfer functions. The mathematical modeling using the FAM approach provides leverage to the designer to comfortably adjust their design at any stage of analysis. These statements provide compelling reasons for the adoption of the proposed process and demonstrate its benefits.
The theoretically obtained equations meet the expected result for the $\mathrm{RC}$ bridge- $\mathrm{T}$ network. Its response peaks at the theoretically obtained value of the frequency. The simulated results are in agreement with the topological explanations and expectations.

Keywords: Band-Pass Filters, Active Filters, Passive Filters, Mathematical model, Floating Admittance Matrix.

\section{INTRODUCTION}

THOUGH the importance of RC bridge-T network in the field of radio frequency applications is very well known, yet it received very little attention. The analysis of the circuit is based on the transfer impedances of the components of the circuit. The selectivity and the shift of the RC bridge-T network can be easily controlled by variation of the circuit parameters. In view of the above statements, the RC bridge$\mathrm{T}$ network are used as wave filters having the desired frequency response characteristic over the selected band of frequencies and also as feedback circuits in amplifiers. Active and passive filters are building blocks of any electronic and communication systems that can alter or reshape the amplitude and/or phase characteristics of a signal. Ideally, active and passive filter alters the various frequency components associated with amplitudes and the phase. 
The frequency-domain behavior of signals defines a particular type of filter. The transfer function of any filter is simply the Laplace transform of the ratio of output signal to the input signal. The filter circuits consisting of any active devices (transistors) and/or Op. amps, in addition to resistors, inductors, and capacitors, are called the active filter. On the other hand, a filter circuits designed using only passive components such as resistors, capacitors, and inductors, are called passive filters. The operating frequency range of the filter is used to determine the electronic components in designing the circuit. Hence, the filter is further categorized based on the operating frequency range. In signal processing, a band-stop filter or band-reject or notch filter are circuits that pass almost all frequencies unchanged but attenuates those in a specific range to very low levels. It is working just opposite to a band pass filter. The notch filter is a special subset of the band-stop filter designed to stop only one frequency or a very small band of frequencies. It is a band-stop filter with a very narrow stop-band. Other names of this type of filters are "Tnotch filter," "band limit filter," "band-elimination filter," and "band-reject filter."

\section{METHODOLOGY}

The conventional method of analysis uses one of the prevalent methods from among KCL, KVL, Thevenin's, Norton's, etc., as per the suitability for that particular circuit, whether active or passive. The proposed floating admittance matrix method is unique, and the same can be used for all types of circuits. The complicated network utilizes the advantage of the matrix partitioning technique. The sum property of all elements of any row or any column equal to zero provides confidence to proceed further for analysis or reobserve the circuit at the very first equation to save time and energy.

The planar spiral type of inductor occupies more space and is associated with the low-quality factor; which is detrimental for the filter circuit design. For this reason, Faruqe et al. [1] used simulated inductance to achieve the high-quality factor inductor for RF applications. This paper presents a comparative analysis of active inductor design for high-quality factors at high-frequency applications. Active inductor-based circuits are commonly used in integrated circuits where the inductor's quality factor dominates the performance of the designed circuits. As the planner spiral inductor occupies a large area and shows a very low-quality factor, an active inductor is an excellent option for filters to overcome the drawbacks. This paper summarizes the analysis and simulation results to select one of the best active inductor topologies to generate high-quality factors for high-frequency applications in filters.

The use of shunt filters for better system performance was discussed by Shady et al. [2] in the multi constraints belonging to the domain of multi-objective functions. The main aim the paper was to minimize total harmonic distortion of the load voltage, supply line, and cost minimization.

Shady et al. [3] demonstrated the optimal design technique to effectively utilize the cables and transformers for harmonically contaminated voltages and currents, accounting for the frequency-dependent loss of power. The method is, especially, suitable for a higher current carrying capacity of load. It is known that HP filters have dual properties of the
LP filters in the sense of sensitivity. Among various topologies of the BP filters, one of the best topology was demonstrated in this article.

The knowledge of the transfer function's characterization gives enough information to decide that the correct functioning of the circuit will be achieved. The variations or changes in the input and output impedances, power supply coupling and uncoupling, variation in the circuit components, and the other dynamic behaviors in the filters' structure are essential parameters discussed in detail by Bogdan [4].

Sargar et al. [5] suggested in-depth modeling and design procedure of LCR filter, primarily for inverter design that suites very well the alternative green energy sources such as a solar system. The MATLAB simulation of PV cell, DC-DC boost converter, and inverter with LCLR filter was also highlighted in the paper.

The heuristic method is supposed to be the easiest and the best for the single tuned passive filters' optimized solution with tapping of its essential parameters; which are very difficult. Such techniques provide a reasonable solution in a short time as it uses fewer iterations. The process called Response Surface Methodology (RSM) proposed by Sakar et al [6] was presented to solve issues of paper [5]. This approach minimize the harmonic distortion in voltage and current of the circuit.

Mathematical modeling based on the admittance matrix model [7]-[9] used older elements such as norators, and nullators. The concepts of nodal equations for filter circuits are available at length in books [10]-[12]. The history of network synthesis and filter theory for circuits consisting of resistors, inductors, and capacitors and low sensitivity sab band-pass active- $R C$ filters using impedance tapering were presented in [13]-[14]. The desensitization using impedance tapering was used to design a class-3 circuit with negative feedback [14]. In the negative feedback loops, the RC-section impedance was scaled upward from the driving source to the negative terminal of the amplifier's input. Paper [15] suggested modeling techniques for tapping different parameters of FETs and BJTs using FAM approach. The expression for voltage gain was derived using FAM in [15][19].

Frequency-selective circuits pass the signals of a range of frequencies through it without alteration in the input signal's magnitude to the output port. However, the magnitude of the input signal passed is reduced drastically, ideally to zero value, outside the pass band. The input current of such circuits are of no significance and hence current transfer functions of such circuits are normally not examined. In the subsequent sections and subsection, we will take up the analysis of RC bridge-T networks of the type-1 and type- 2 using the floating admittance matrix approach (FAM) and its plots. 


\section{CONVENTIONAL ANALYSIS OF RC BRIDGE-T NETWORK}

The conventional KVL method of analysis of type-1 RC bridge-T network in Fig. 1 runs into many equations as below.

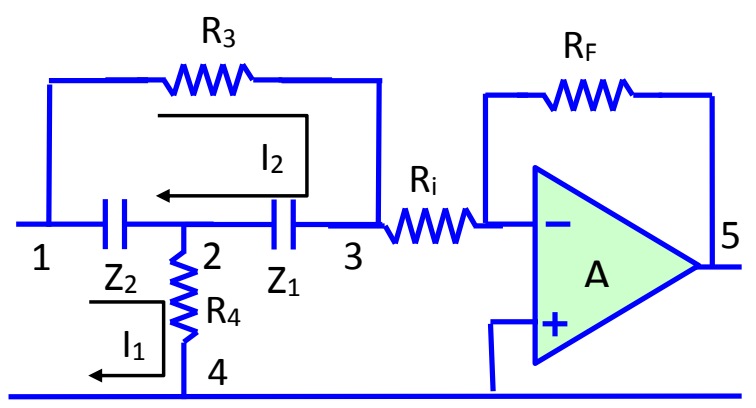

Fig. 1 Type-1 RC bridge-T network

$V_{3}=V_{1}-I_{2} R_{3}$

$V_{1}-I_{1}\left(Z_{2}+R_{4}\right)+I_{2} Z_{2}=0$

$I_{1} Z_{2}-I_{2}\left(R_{3}+Z_{1}+Z_{2}\right)=0$

$I_{1}=I_{2} \frac{R_{3}+Z_{1}+Z_{2}}{Z_{2}}$

$V_{1}-I_{2} \frac{R_{3}+Z_{1}+Z_{2}}{Z_{2}}\left(Z_{2}+R_{4}\right)+I_{2} Z_{2}=0$

$V_{1}-I_{2}\left(Z_{1}+R_{3}\right)-\left(R_{3}+Z_{1}+Z_{2}\right) \frac{R_{4}}{Z_{2}} I_{2}=0$

$V_{1}-I_{2}\left(Z_{1}+R_{3}\right)-\left(R_{4}+R_{3} \frac{R_{4}}{Z_{2}}+\frac{R_{4}}{Z_{2}} Z_{1}\right) I_{2}=0$

$V_{1}-I_{2}\left(Z_{1}+R_{3}+R_{4}\right)-\left(R_{3} \frac{R_{4}}{Z_{2}}+\frac{R_{4}}{Z_{2}} Z_{1}\right) I_{2}=0$

$I_{2}=\frac{V_{1}}{Z_{1}+R_{3}+R_{4}+\frac{R_{3} R_{4}}{Z_{2} R_{3}}+\frac{R_{4} Z_{1}}{Z_{2} R_{3}}}$

$I_{2} R_{3}=\frac{V_{1}}{1+\frac{R_{4}}{R_{3}}+\frac{Z_{1}}{R_{3}}+\frac{R_{4} R_{3}}{Z_{2} R_{3}}+\frac{Z_{1} R_{4}}{Z_{2} R_{3}}}=\frac{V_{1}}{1+\frac{Z_{1}}{R_{3}}+\frac{R_{4}}{R_{3}}+\frac{R_{4}}{Z_{2}}+\frac{Z_{1} R_{4}}{Z_{2} R_{3}}}$

$I_{2} R_{3}=\frac{V_{1}}{1+\frac{R_{4}}{R_{3}}\left(1+\frac{Z_{1}}{Z_{2}}\right)+\frac{Z_{1}}{R_{3}}\left(1+\frac{R_{4} R_{3}}{Z_{2} Z_{1}}\right)}$

$I_{2} R_{3}=\frac{V_{1}}{1+\frac{R_{4}}{R_{3}}\left(1+\frac{\frac{1}{s C_{1}}}{\frac{1}{s C_{2}}}\right)+\frac{1}{s C_{1}}\left\{1+\frac{R_{3} R_{4}}{R_{3}}\left\{\begin{array}{c}\frac{1}{s C_{2}} \cdot \frac{1}{s C_{1}} \\ V_{1}\end{array}\right\}\right.}$

$=\frac{V_{1}}{1+\frac{R_{4}}{R_{3}}\left(1+\frac{C_{2}}{C_{1}}\right)+\frac{1}{s C_{1} R_{3}}\left\{1+s^{2} C_{1} C_{2} R_{4} R_{3}\right\}}$

$\frac{V_{3}}{V_{1}}=1-\frac{I_{2} R_{3}}{V_{1}}=\frac{s^{2} C^{2} R_{4} R_{3}+2 s C R_{4}+1}{s^{2} C^{2} R_{4} R_{3}+s C\left(R_{3}+2 R_{4}\right)+1}$

$\frac{V_{3}}{V_{1}}=\frac{s^{2}+s \frac{2}{C R_{3}}+\frac{1}{C^{2} R_{4} R_{3}}}{s^{2}+s \frac{\left(R_{3}+2 R_{4}\right)}{C R_{4} R_{3}}+\frac{1}{C^{2} R_{4} R_{3}}}$

$=\frac{s^{2}+s \frac{2}{C R_{3}}+\omega_{o}^{2}}{s^{2}+s \frac{\omega_{O}}{Q}+\omega_{o}^{2}}=\frac{s^{2}+s \frac{\omega_{O 1}}{Q_{1}}+\omega_{o}^{2}}{s^{2}+s \frac{\omega_{O}}{Q}+\omega_{o}^{2}}$

$=\frac{s^{2}+s \frac{2}{C R_{3}}+\omega_{o}^{2}}{s^{2}+2 s \delta \omega_{o}+\omega_{o}^{2}}$

$2 \delta \omega_{o}=\frac{\left(R_{3}+2 R_{4}\right)}{C R_{4} R_{3}}, \omega_{o}=\frac{1}{C \sqrt{R_{4} R_{3}}}$

$\delta=\frac{\left(R_{3}+2 R_{4}\right)}{C R_{4} R_{3}} x \frac{1}{2 \omega_{0}}=\frac{\left(R_{3}+2 R_{4}\right)}{C R_{4} R_{3}} x \frac{C \sqrt{R_{4} R_{3}}}{2}$

$=\frac{\left(R_{3}+2 R_{4}\right)}{\sqrt{R_{4} R_{3}}} x \frac{1}{2}=\left(\frac{1}{2} \sqrt{\frac{R_{3}}{R_{4}}}+\sqrt{\frac{R_{4}}{R_{3}}}\right)$
This is the general equation of a filter transfer function.

Where, $\omega_{o}=\frac{1}{\sqrt{C^{2} R_{3} R_{4}}}, Q=\frac{\sqrt{R_{3} R_{4}}}{R_{3}+2 R_{4}}$

For symmetrical RC bridge-T filter $R_{3}=R_{4}=R$ then,

$$
\omega_{o}=\frac{1}{C R}
$$

Similar analysis is performed on type-2 RC bridge-T network that yields the same type of result.

\section{TYPE-1 RC BRIDGE T-NETWORK ANALYSIS USING FAM}

The floating admittance matrix of the type-1 RC bridge- $\mathrm{T}$ network without Op. Amp in Fig. 1 is written [15]-[19] as;

$$
\left[\begin{array}{cccc}
1 & 2 & 3 & 4 \\
G_{3}+s C_{2} & -s C_{2} & -G_{3} & 0 \\
-s C_{2} & s C_{1}+s C_{2}+G_{4} & -s C_{1} & -G_{4} \\
-G_{3} & -s C_{1} & G_{3}+s C_{1} & 0 \\
0 & -G_{4} & 0 & G_{4}
\end{array}\right] \begin{aligned}
& 2 \\
& 4
\end{aligned}
$$

The open-circuit voltage transfer function between terminals $3 \& 4$ and $1 \& 4$ can be expressed [15]-[19] as;

$$
\begin{aligned}
& \left.A_{v}\right|_{14} ^{34}=\operatorname{sgn}(3-4) \operatorname{sgn}(1-4)(-1)^{12} \frac{\left|Y_{34}^{14}\right|}{\left|Y_{14}^{14}\right|} \\
& \left|Y_{34}^{14}\right|=\left|\begin{array}{cc}
-s C_{2} & s C_{1}+s C_{2}+G_{4} \\
-G_{3} & -s C_{1}
\end{array}\right|=s^{2}+s \frac{\left(C_{1}+C_{2}\right) G_{3}}{C_{1} C_{2}}+\frac{G_{3} G_{4}}{C_{1} C_{2}} \\
& \left|Y_{14}^{14}\right|=\left|\begin{array}{cc}
s C_{1}+s C_{2}+G_{4} & -s C_{1} \\
-s C_{1} & G_{3}+s C_{1}
\end{array}\right| \\
& =\left|\begin{array}{cc}
s C_{2}+G_{4} & -s C_{1} \\
G_{3} & G_{3}+s C_{1}
\end{array}\right|=\left|\begin{array}{cc}
s C_{2}+G_{3}+G_{4} & G_{3} \\
G_{3} & G_{3}+s C_{1}
\end{array}\right| \\
& =s^{2}+s \frac{\left\{\left(C_{2}+C_{1}\right) G_{3}+C_{1} G_{4}\right\}}{C_{1} C_{2}}+\frac{G_{3} G_{4}}{C_{1} C_{2}} \\
& \left.A_{v}\right|_{14} ^{34}=\frac{s^{2}+s\left(\frac{1}{C_{1}}+\frac{1}{C_{2}}\right) \frac{1}{R_{3}}+\frac{1}{C_{1} C_{2} R_{3} R_{4}}}{s^{2}+s\left\{\left(\frac{1}{C_{1}}+\frac{1}{C_{2}}\right) \frac{1}{R_{3}}+\frac{1}{C_{2} R_{4}}\right\}+\frac{1}{C_{1} C_{2} R_{3} R_{4}}} \\
& =\frac{s^{2}+s a+\omega_{0}^{2}}{s^{2}+s b+\omega_{0}^{2}}=\frac{s^{2}+s \frac{\omega_{0}}{Q}+\omega_{0}^{2}}{s^{2}+s \frac{\omega_{01}}{Q}+\omega_{0}^{2}}
\end{aligned}
$$

Since, $\left.\beta(s)\right|_{34} ^{14}=\frac{1}{\left.A_{v}\right|_{14} ^{34}}$

Figure 1 is the circuit of type-1 RC bridge-T network including the inverting Op Amp. The overall voltage transfer function of Fig. 1 is;

$$
\begin{aligned}
\left.A_{v}\right|_{14} ^{54} & =\frac{v_{54}}{v_{14}}=\frac{v_{54}}{v_{34}} x \frac{v_{34}}{v_{14}} \\
& =\frac{s^{2}+s \frac{\omega_{0}}{Q}+\omega_{0}^{2}}{s^{2}+s \frac{\omega_{0}}{Q_{1}}+\omega_{0}^{2}} x\left(-\frac{R_{F}}{R_{i}}\right)=-\frac{s^{2}+s \frac{\omega_{0}}{Q}+\omega_{0}^{2}}{s^{2}+s \frac{\omega_{0}}{Q_{1}}+\omega_{0}^{2}} \text { for } \mathrm{R}_{\mathrm{F}}=\mathrm{R}_{\mathrm{i}}
\end{aligned}
$$

The transmission zeros in Eq. (14) are similar to the poles of the closed-loop circuit in Fig. 1. Hence, the transmission zeros form the polynomial as;

$$
\begin{aligned}
& s^{2}+s \frac{\omega_{0}}{Q}+\omega_{O}^{2}=s^{2}+s a+\omega_{O}^{2} \\
& =s^{2}+s\left(\frac{1}{C_{1}}+\frac{1}{C_{2}}\right) \frac{1}{R_{3}}+\frac{1}{C_{1} C_{2} R_{3} R_{4}}
\end{aligned}
$$


$\omega_{0}=\frac{1}{\sqrt{C_{1} C_{2} R_{3} R_{4}}}$

$Q=\frac{\omega_{0}}{a}=\frac{\omega_{0}}{\left(\frac{1}{C_{1}}+\frac{1}{C_{2}}\right) \frac{1}{R_{3}}}=\frac{1}{\sqrt{C_{1} C_{2} R_{3} R_{4}}} x \frac{1}{\left\{\frac{C_{1}+C_{2}}{C_{1} C_{2} R_{3}}\right\}}=\frac{\sqrt{C_{1} C_{2} R_{3} / R_{4}}}{C_{1}+C_{2}}$

For, $\mathrm{R}_{2}=\mathrm{R}_{4}=\mathrm{R}$, and $\mathrm{C}_{1}=\mathrm{C}_{2}=\mathrm{C}, \mathrm{Q}=0.5$

The characteristic equation for the poles is;

$s^{2}+s \frac{\omega_{0}}{Q_{1}}+\omega_{0}^{2}=s^{2}+s b+\omega_{O}^{2}$

where, $b=\left\{\left(\frac{1}{C_{1}}+\frac{1}{C_{2}}\right) \frac{1}{R_{3}}+\frac{1}{C_{2} R_{4}}\right\}=\frac{2}{R C}+\frac{1}{R C}=\frac{3}{R C}$, and

$\omega_{0}=\frac{1}{R C}, \frac{\omega_{0}}{Q_{1}}=\frac{3}{R C}=3 \omega_{0}, Q_{1}=\frac{1}{3}$

The simplified characteristic equation for the pole is now expressed as;

$$
s^{2}+3 \omega_{0} s+\omega_{0}^{2}
$$

As stated above, though there is no need to find out the current transfer ratio for such networks, we include here both, current transfer and power transfer ratios for academic purposes. The current transfer function between terminals $3 \& 4$ and $1 \& 4$ can be expressed [15]-[17] as;

$\left.A_{i}\right|_{14} ^{34}=\operatorname{sgn}(3-4) \operatorname{sgn}(1-4)(-1)^{12} \frac{\left|Y_{34}^{14}\right|}{\left|Y_{4}^{4}\right|} G_{L}$

$\left|Y_{4}^{4}\right|=\left|\begin{array}{ccc}G_{3}+s C_{2} & -s C_{2} & -G_{3} \\ -s C_{2} & s C_{1}+s C_{2}+G_{4} & -s C_{1} \\ -G_{3} & -s C_{1} & G_{3}+s C_{1}\end{array}\right|$

$=\left|\begin{array}{ccc}G_{3}+s C_{2} & 0 & -G_{3} \\ 0 & G_{4} & 0 \\ -G_{3} & 0 & G_{3}+s C_{1}\end{array}\right|$

$=G_{4} s\left\{s C_{1} C_{2}+G_{3}\left(C_{1}+C_{2}\right)\right\}$

$\left.A_{i}\right|_{14} ^{34}=\frac{s^{2} C_{1} C_{2}+s\left(C_{1}+C_{2}\right) G_{3}+G_{3} G_{4}}{G_{4} s\left\{s C_{1} C_{2}+G_{3}\left(C_{1}+C_{2}\right)\right\}} G_{L}$

The power transfer function between terminals $3 \& 4$ and $1 \&$ 4 can be expressed as;

$\left.A_{p}\right|_{14} ^{34}=\left.\left.A_{v}\right|_{14} ^{34} x A_{i}\right|_{14} ^{34}$

$\left.A_{p}\right|_{14} ^{34}=\left\{\frac{s^{2} C_{1} C_{2}+s\left(C_{1}+C_{2}\right) G_{3}+G_{3} G_{4}}{s^{2} C_{1} C_{2}+s\left\{\left(C_{2}+C_{1}\right) G_{3}+C_{1} G_{4}\right\}+G_{3} G_{4}}\right\} x$ $\left\{\frac{s^{2} C_{1} C_{2}+s\left(C_{1}+C_{2}\right) G_{3}+G_{3} G_{4}}{G_{4} s\left\{s C_{1} C_{2}+G_{3}\left(C_{1}+C_{2}\right)\right\}} G_{L}\right\}$

\section{A. Experimental Verification}

The MATLAB program for the type-1 RC bridge-T network is given below and its Bode plot is shown in Fig. 2. It depicts the plot for amplitude and phase variation with reference to frequency. The assumed value of resistances and capacitances are indicated in the MATLAB program below.

r3 $=1500 ; \mathrm{r} 4=1500$;

$\mathrm{c} 1=1000 \mathrm{e}-6 ; \mathrm{c} 2=2000 \mathrm{e}-6$;

$\mathrm{A}=(1 / \mathrm{c} 1+1 / \mathrm{c} 2) / \mathrm{r} 3$

$\mathrm{B}=1 / \mathrm{c} 1 * 1 / \mathrm{c} 2 * 1 / \mathrm{r} 3 * 1 / \mathrm{r} 4$

$\mathrm{C}=1 / \mathrm{c} 1 * 1 / \mathrm{c} 2 * 1 / \mathrm{r} 3 * 1 / \mathrm{r} 4$

$\mathrm{D}=1 / \mathrm{c} 1 * 1 / \mathrm{c} 2 * 1 / \mathrm{r} 3 * 1 / \mathrm{r} 4$

num $=\left[\begin{array}{lll}1 & \mathrm{~A} & \mathrm{~B}\end{array}\right]$;

den $=[1 \mathrm{C} \mathrm{D}]$

$\mathrm{GP}=\mathrm{tf}($ num,den $)$

bode(GP);

$$
\mathrm{GP}=\frac{\mathrm{s}^{\wedge} 2+\mathrm{s}+0.2222}{-\mathrm{s}^{\wedge} 2+0.2222 \mathrm{~s}+0.2222}
$$

The plot of the above-described continuous time domain voltage transfer function without and with an Op. Amp inverter are shown in Figs 2 and 3, respectively, which indicate phase difference of 180 degrees, as expected with Op Amp inverter.

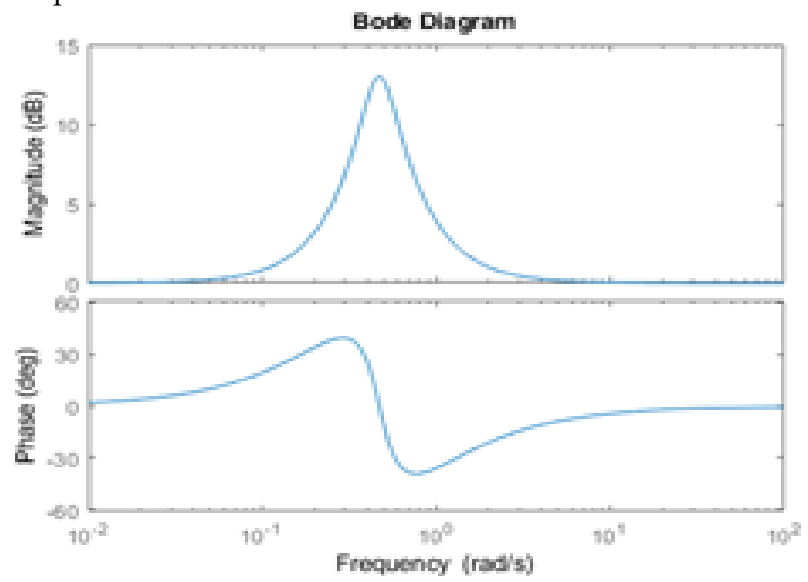

Fig. 2 Plot of type-1 RC bridge-T network transfer function w.r.t. frequency without inclusion of Op. Amp inverter

The MATLAB program for the type-1 RC bridge-T network in Fig. 1 and its Bode plot including Op Amp inverter is shown in Fig. 3.

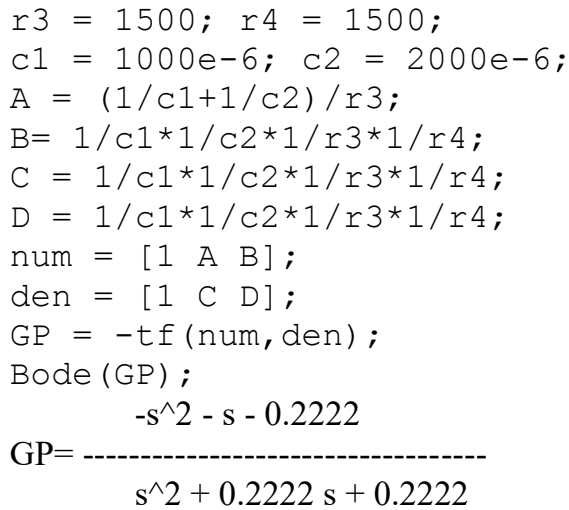

This is the continuous-time domain transfer function.

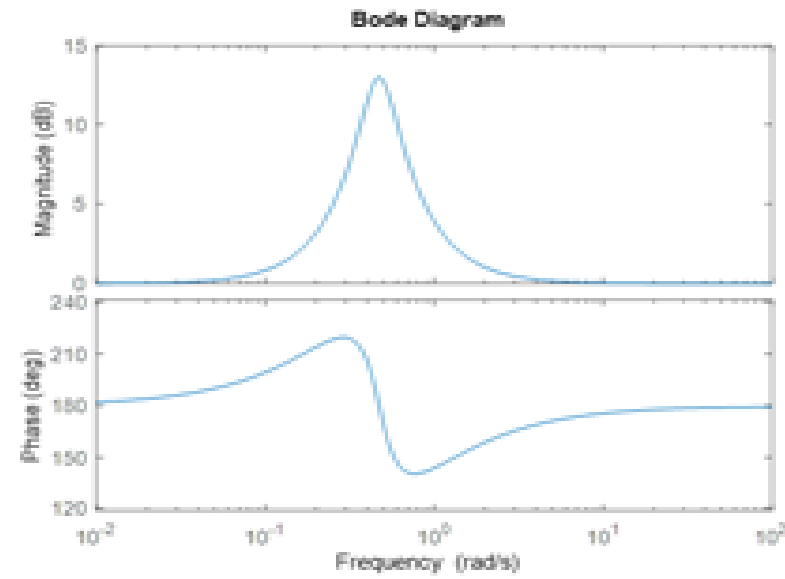

Fig. 3 Plot of type-1 RC bridge-T network including Op. Amp inverter w.r.t. frequency 
The input impedance between terminals $1 \& 4$ in Fig. 1 is expressed [15]-[17] as;

$Z_{\text {in }}=Z_{14}=\frac{\left|Y_{14}^{14}\right|}{\left|Y_{4}^{4}\right|} g_{s=0}$

$\left|Y_{4}^{4}\right|=\left|\begin{array}{ccc}G_{3}+s C_{2} & -s C_{2} & -G_{3} \\ -s C_{2} & s C_{1}+s C_{2}+G_{4} & -s C_{1} \\ -G_{3} & -s C_{1} & G_{3}+s C_{1}\end{array}\right|$

$=\left|\begin{array}{ccc}G_{3}+s C_{2} & 0 & -G_{3} \\ -s C_{2} & G_{4} & -s C_{1} \\ -G_{3} & 0 & G_{3}+s C_{1}\end{array}\right|=G_{4}\left|\begin{array}{cc}G_{3}+s C_{2} & -G_{3} \\ -G_{3} & G_{3}+s C_{1}\end{array}\right|$

$=G_{4}\left|\begin{array}{cc}s C_{2} & -G_{3} \\ s C_{1} & G_{3}+s C_{1}\end{array}\right|$

$=G_{4} s\left\{s C_{1} C_{2}+\left(C_{1}+C_{2}\right) G_{3}\right\}$

$Z_{\text {in }}=Z_{14}=\frac{s^{2} C_{1} C_{2}+s\left\{\left(C_{1}+C_{2}\right) G_{3}+C_{1} G_{4}\right\}+G_{3} G_{4}}{G_{4} s\left\{s C_{1} C_{2}+\left(C_{1}+C_{2}\right) G_{3}\right\}}$

$=\frac{1}{G_{4}}+\frac{s C_{1}+G_{3}}{s C_{2}\left(s C_{1}+G_{3}\right)+C_{1} G_{3}}$

$=\frac{1}{G_{4}}+\frac{1}{s C_{2}+\frac{s C_{1} G_{3}}{s C_{1}+G_{3}}}$

This Eq. (26) of input impedance represents the network of the form shown in Fig. 4.

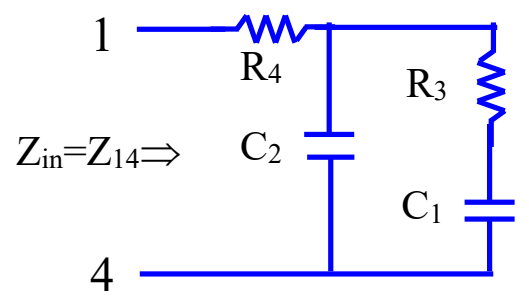

Fig. 4 input impedance representation

The output impedance between terminals $3 \& 4$ of Fig. 1 can be expressed [15]-[17] as;

$$
\begin{gathered}
R_{o}=R_{34}=\frac{\left|Y_{34}^{34}\right|}{\left|Y_{4}^{4}\right|} G_{L}=0 \\
\left|Y_{34}^{34}\right|=\left|\begin{array}{cc}
G_{3}+s C_{2} & -s C_{2} \\
-s C_{2} & s C_{1}+s C_{2}+G_{4}
\end{array}\right| \\
=\left|\begin{array}{cc}
G_{3}+s C_{2} & G_{3} \\
-s C_{2} & s C_{1}+G_{4}
\end{array}\right| \\
=s^{2} C_{1} C_{2}+s\left(C_{1}+C_{2}\right) G_{3}+s C_{2} G_{4}+G_{3} G_{4} \\
R_{o}=R_{34}=\frac{\left|Y_{34}^{34}\right|}{\left|Y_{4}^{4}\right|} G_{L}=0 \\
=\frac{s^{2} C_{1} C_{2}+s\left(C_{1}+C_{2}\right) G_{3}+s C_{2} G_{4}+G_{3} G_{4}}{G_{4}\left\{s^{2} C_{1} C_{2}+s\left(C_{1}+C_{2}\right) G_{3}\right\}} \\
=\frac{1}{G_{4}}+\frac{\left(s C_{2}+G_{3}\right)}{s\left(s C_{2}+G_{3}\right) C_{1}+s C_{2} G_{3}}=\frac{1}{G_{4}}+\frac{1}{s C_{1}+\frac{s C_{2} G_{3}}{s C_{2}+G_{3}}}
\end{gathered}
$$

The circuit of output impedance in Fig. 5 represents Eq. (29).

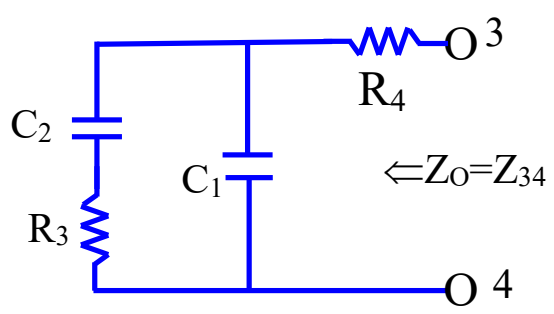

Fig.5 Output impedance representation

\section{TYPE-2 RC BRIDGE T-NETWORK ANALYSIS USING FAM}

Figure 6 shows the dual (type-2) form of the RC bridge-T network for realizing the same type of transmission zeros and poles.

The floating admittance matrix for Fig. 6 is expressed [15][19] as;

$\left[\begin{array}{cccc}1 & 2 & 3 & 4 \\ G_{2}+s C_{3} & -G_{2} & -s C_{3} & 0 \\ -G_{2} & G_{1}+G_{2}+s C_{4} & -G_{1} & -s C_{4} \\ -s C_{3} & -G_{1} & G_{1}+s C_{3} & 0 \\ 0 & -s C_{4} & 0 & s C_{4}\end{array}\right] \begin{aligned} & 2 \\ & 4\end{aligned}$

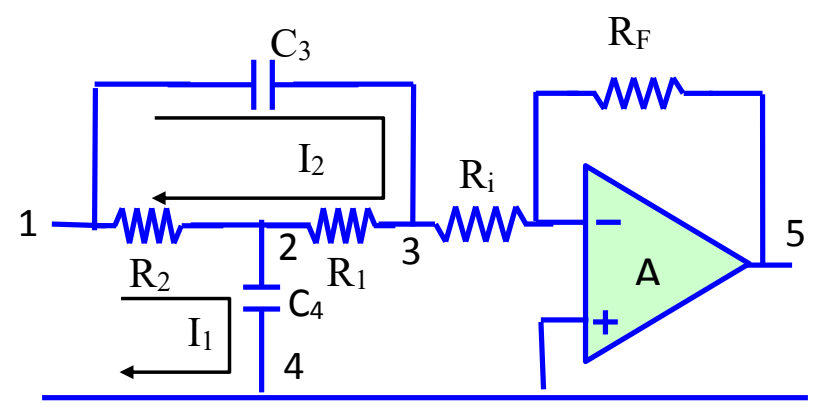

Fig. 6 Type-2 RC bridge-T network

The open-circuit voltage transfer function between terminals $3 \& 4$ and $1 \& 4$ of Fig. 6 can be expressed [15]-[19] as;

$$
\begin{aligned}
& \left.A_{v}\right|_{14} ^{34}=\operatorname{sgn}(3-4) \operatorname{sgn}(1-4)(-1)^{12} \frac{\left|Y_{34}^{14}\right|}{\left|Y_{14}^{14}\right|} \\
& \left|Y_{34}^{14}\right|=\left|\begin{array}{cc}
-G_{2} & G_{1}+G_{2}+s C_{4} \\
-s C_{3} & -G_{1}
\end{array}\right| \\
& =G_{1} G_{2}+s\left(G_{1}+G_{2}\right) C_{3}+s^{2} C_{3} C_{4} \\
& =s^{2}+s \frac{\left(G_{1}+G_{2}\right) C_{3}}{C_{3} C_{4}}+\frac{G_{1} G_{2}}{C_{3} C_{4}} \\
& \left|Y_{14}^{14}\right|=\left|\begin{array}{cc}
G_{1}+G_{2}+s C_{4} & -G_{1} \\
-G_{1} & G_{1}+s C_{3}
\end{array}\right| \\
& =\left|\begin{array}{cc}
G_{2}+s C_{4} & -G_{1} \\
s C_{3} & G_{1}+s C_{3}
\end{array}\right| \\
& =s^{2} C_{3} C_{4}+s\left\{C_{3}\left(G_{1}+G_{2}\right)+C_{4} G_{1}\right\}+G_{1} G_{2} .
\end{aligned}
$$

$$
\begin{aligned}
& \left.A_{v}\right|_{14} ^{34}=\frac{\left|Y_{34}^{14}\right|}{\left|Y_{14}^{14}\right|}=\frac{s^{2}+s \frac{\left(G_{1}+G_{2}\right) C_{3}}{C_{3} C_{4}}+\frac{G_{1} G_{2}}{C_{3} C_{4}}}{s^{2}+s \frac{\left\{C_{3}\left(G_{1}+G_{2}\right)+C_{4} G_{1}\right\}}{C_{3} C_{4}}+\frac{G_{1} G_{2}}{C_{3} C_{4}}} \\
& =\frac{s^{2}+s\left(\frac{1}{R_{1}}+\frac{1}{R_{2}}\right) \frac{1}{C_{4}}+\frac{1}{C_{3} C_{4} R_{1} R_{2}}}{s^{2}+s\left\{\left(\frac{1}{R_{1}}+\frac{1}{R_{2}}\right) \frac{1}{C_{4}}+\frac{1}{R_{1} C_{3}}\right\}+\frac{1}{C_{3} C_{4} R_{1} R_{2}}}=\frac{s^{2}+s \frac{\omega_{0}}{Q}+\omega_{0}^{2}}{s^{2}+s \frac{\omega_{0}}{Q_{1}}+\omega_{0}^{2}}
\end{aligned}
$$


The overall voltage transfer function between terminals $5 \&$ 4 and $1 \& 4$ including the Op. Amp is;

$$
\begin{aligned}
\left.A_{v}\right|_{14} ^{54} & =\frac{v_{54}}{v_{14}}=\frac{v_{34}}{v_{14}} x \frac{v_{54}}{v_{34}}=\frac{s^{2}+s \frac{\omega_{0}}{Q}+\omega_{0}^{2}}{s^{2}+s \frac{\omega_{0}}{Q_{1}}+\omega_{0}^{2}} x\left(-\frac{R_{F}}{R_{i}}\right) \\
& =\frac{s^{2}+s \frac{\omega_{0}}{Q}+\omega_{0}^{2}}{s^{2}+s \frac{\omega_{0}}{Q_{1}}+\omega_{0}^{2}} \text { for } R_{i}=R_{F}
\end{aligned}
$$

In Eq. (33), the transmission zero is similar to the poles of the closed-loop circuit of Fig. 6. Hence, the transmission zero is expressed as;

$s^{2}+s \frac{\omega_{0}}{Q}+\omega_{0}^{2}=s^{2}+a s+\omega_{0}^{2}$

$=s^{2}+s\left(\frac{1}{R_{1}}+\frac{1}{R_{2}}\right) \frac{1}{C_{4}}+\frac{1}{C_{3} C_{4} R_{1} R_{2}}$

here, $\omega_{O}=\frac{1}{\sqrt{C_{3} C_{4} R_{1} R_{2}}}$ and

$Q=\frac{\omega_{0}}{a}=\sqrt{\frac{1}{C_{3} C_{4} R_{1} R_{2}}} x \frac{1}{\frac{\left(G_{1}+G_{2}\right)}{C_{4}}}=\frac{\sqrt{R_{1} R_{2} C_{4} / C_{3}}}{R_{1}+R_{2}}$

For, $\mathrm{C}_{3}=\mathrm{C}_{4}=\mathrm{C}$, and $\mathrm{R}_{1}=\mathrm{R}_{2}=\mathrm{R}, \omega_{\mathrm{o}}=1 / \mathrm{RC}, \mathrm{Q}=0.5$

The characteristic equation for poles in Eq. (21) is;

$$
s^{2}+s \frac{\omega_{0}}{Q_{1}}+\omega_{0}^{2}=s^{2}+b s+\omega_{0}^{2},
$$

where, $b=\left(\frac{1}{R_{1}}+\frac{1}{R_{2}}\right) \frac{1}{C_{4}}+\frac{1}{R_{1} C_{3}}=\frac{3}{R C}$,

$\frac{\omega_{0}}{Q_{1}}=\frac{3}{R C}=3 \omega_{0}, Q_{1}=\frac{1}{3}$

The characteristic equation for poles is now expressed as;

$$
s^{2}+3 \omega_{0} s+\omega_{0}^{2}
$$

The current transfer function between terminals $3 \& 4$ and 1 $\& 4$ of Fig. 6 can be expressed [15] - [17] as;

$$
\begin{aligned}
& \left.A_{i}\right|_{14} ^{34}=\operatorname{sgn}(3-4) \operatorname{sgn}(1-4)(-1)^{12} \frac{\left|Y_{34}^{14}\right|}{\left|Y_{4}^{4}\right|} G_{L} \\
& \left|Y_{4}^{4}\right|=\left|\begin{array}{ccc}
G_{2}+s C_{3} & -G_{2} & -s C_{3} \\
-G_{2} & G_{1}+G_{2}+s C_{4} & -G_{1} \\
-s C_{3} & -G_{1} & G_{1}+s C_{3}
\end{array}\right| \\
& =s C_{4}\left|\begin{array}{cc}
G_{2}+s C_{3} & -s C_{3} \\
-s C_{3} & G_{1}+s C_{3}
\end{array}\right|=s C_{4}\left|\begin{array}{cc}
G_{2} & -s C_{3} \\
G_{1} & G_{1}+s C_{1}
\end{array}\right| \\
& =s C_{4}\left\{s C_{1} G_{2}+s C_{3} G_{1}+G_{1} G_{3}\right\} \\
& \left.A_{i}\right|_{14} ^{34}=\frac{\left|Y_{34}^{14}\right|}{\left|Y_{4}^{4}\right|} G_{L}=\frac{s^{2} C_{3} C_{4}+s\left(G_{1}+G_{2}\right) C_{3}+G_{1} G_{2}}{s C_{4}\left\{s C_{1} G_{2}+s C_{3} G_{1}+G_{1} G_{3}\right\}} G_{L}
\end{aligned}
$$

The power transfer function between terminals $3 \& 4$ and $1 \&$ 4 of Fig. 6 can be expressed as;

$\left.A_{p}\right|_{14} ^{34}=\left.\left.A_{v}\right|_{14} ^{34} x A_{i}\right|_{14} ^{34}$

$=\left\{\frac{s^{2} C_{3} C_{4}+s\left(G_{1}+G_{2}\right) C_{3}+G_{1} G_{2}}{s^{2} C_{3} C_{4}+s\left\{C_{3}\left(G_{1}+G_{2}\right)+C_{4} G_{1}\right\}+G_{1} G_{2}}\right\}\left\{\frac{s^{2} C_{3} C_{4}+s\left(G_{1}+G_{2}\right) C_{3}+G_{1} G_{2}}{s C_{4}\left\{s C_{1} G_{2}+s C_{3} G_{1}+G_{1} G_{3}\right\}} G_{L}\right\}$

\section{A. Experimental Verification}

The MATLAB program for the type-2 RC bridge-T network shown in Fig. 6 is given below. Its Bode plot is shown in Fig. 7. The plots show amplitude and phase variation with reference to frequency. The assumed value of resistances and capacitances are indicated in the program.

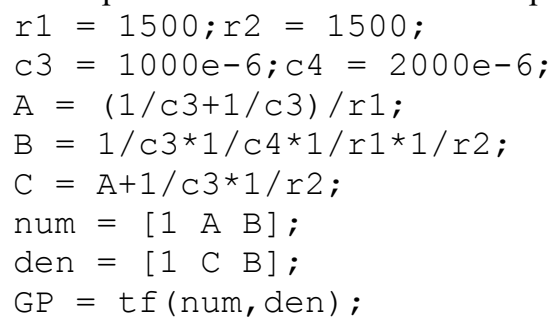

bode (GP) ;

$$
\begin{array}{r}
s^{\wedge} 2+1.333 s+0.2222 \\
G P=--------------- \\
s^{\wedge} 2+2 s+0.2222
\end{array}
$$

Figure 7 is the plot of the continuous-time domain transfer function indicated by above Eq. (40).

The MATLAB program for the type- $2 \mathrm{RC}$ bridge-T network shown in Fig. 6 is given below.

$r 1=1500 ; r 2=1500$;
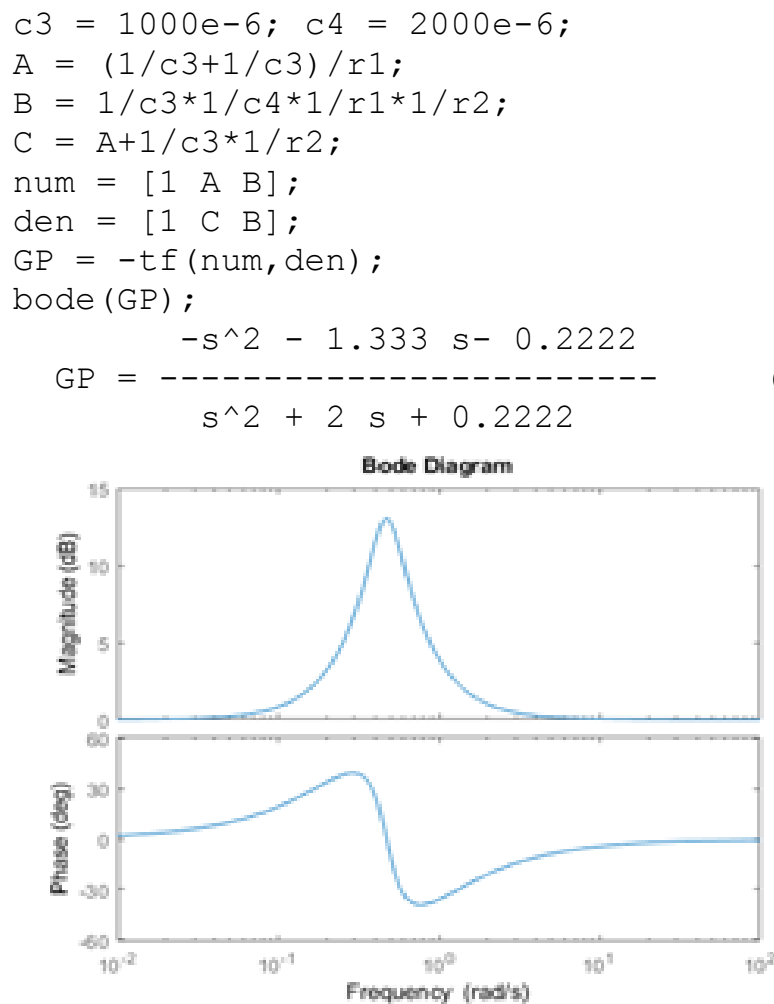

Fig.7 Plot of type-2 RC bridge network transfer function including Op. Amp w.r.t. frequency

The continuous-time domain transfer function indicated by above equation along with Op. Amp inverter in Fig. 6 is plotted in Fig. 8.

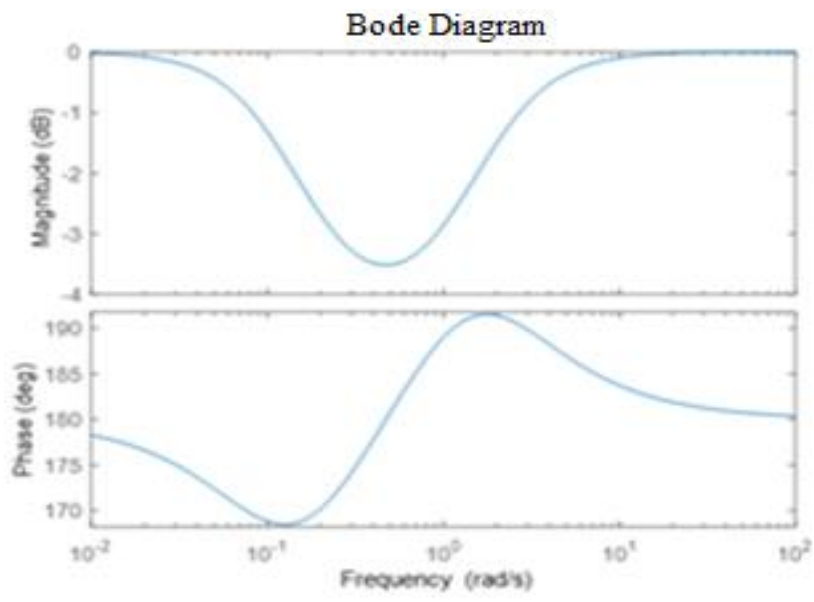

Fig. 8 Magnitude and phase plot of type-2 RC bridge-T network including Op. Amp inverter w.r.t. frequency

The input impedance between terminals $1 \& 4$ of Fig. 6 can be expressed [15]-[17] as;

$$
Z_{\text {in }}=Z_{14}=\frac{\left|Y_{14}^{14}\right|}{\left|Y_{4}^{4}\right|}{ }_{g_{s}=0}
$$




$$
\begin{gathered}
\left|Y_{4}^{4}\right|=\left|\begin{array}{ccc}
G_{2}+s C_{3} & -G_{2} & -s C_{3} \\
-G_{2} & G_{1}+G_{2}+s C_{4} & -G_{1} \\
-s C_{3} & -G_{1} & G_{1}+s C_{3}
\end{array}\right| \\
=\left|\begin{array}{ccc}
0 & s C_{4} & 0 \\
-G_{2} & s C_{4} & -G_{1} \\
-s C_{3} & 0 & G_{1}+s C_{3}
\end{array}\right| \\
=s C_{4}\left\{s\left(G_{1}+G_{2}\right) C_{3}+G_{2} G_{1}\right\} \\
Z_{\text {in }}=Z_{14}=\frac{\left|Y_{14}^{14}\right|}{\left|Y_{4}^{4}\right|}=\frac{s_{s} C_{3} C_{4}+s\left\{C_{3}\left(G_{1}+G_{2}\right)+C_{4} G_{1}\right\}+G_{1} G_{2}}{s C_{4}\left\{s\left(G_{1}+G_{2}\right) C_{3}+G_{2} G_{1}\right\}} \\
=\frac{s\left\{C_{3}\left(G_{1}+G_{2}\right)\right\}+G_{1} G_{2}}{s C_{4}\left\{s\left(G_{1}+G_{2}\right) C_{3}+G_{2} G_{1}\right\}}+\frac{s^{2} C_{3} C_{4}+s C_{4} G_{1}}{s C_{4}\left\{s\left(G_{1}+G_{2}\right) C_{3}+G_{2} G_{1}\right\}} \\
\mathrm{Z}_{i}=\mathrm{Z}_{14} \frac{s^{2} C_{3} C_{4}+s\left\{C_{3}\left(G_{1}+G_{2}\right)+C_{4} G_{1}\right\}+G_{1} G_{2}}{s C_{4}\left\{s\left(G_{1}+G_{2}\right) C_{3}+G_{1} G_{2}\right\}} \\
=\frac{1}{s C_{4}}+\frac{1}{G_{2}+\frac{s C_{3} G_{1}}{s C_{3}+G_{1}}}
\end{gathered}
$$

Figure 9 is the representation of the input impedance of Eq. (44).

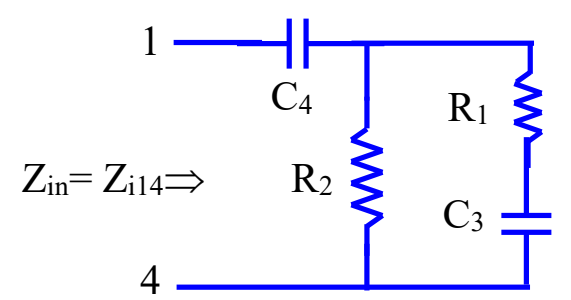

Fig.9 Input impedance representation

The output impedance of Fig. 6 between terminals $3 \& 4$ of type-2 RC bridge-T network can be written [15]-[17] as;

$$
\begin{aligned}
& Z_{O}=Z_{34} \frac{\left|Y_{34}^{34}\right|}{\left|Y_{4}^{4}\right|} G_{L}=0 \\
& \left|Y_{14}^{34}\right|=\left|\begin{array}{cc}
G_{2}+s C_{3} & -G_{2} \\
-G_{2} & G_{1}+G_{2}+s C_{4}
\end{array}\right| \\
& \quad\left|\mathrm{Y}_{34}^{34}\right|=\left|\begin{array}{cc}
\mathrm{G}_{2}+\mathrm{SC}_{3} & -\mathrm{G}_{2} \\
-\mathrm{G}_{2} & \mathrm{G}_{1}+\mathrm{G}_{2}+\mathrm{SC}_{4}
\end{array}\right| \\
& =s^{2} C_{3} C_{4}+s\left\{C_{3}\left(G_{1}+G_{2}\right)+C_{4} G_{2}\right\}+G_{1} G_{2} \\
& Z_{O}=Z_{34}=\frac{s^{2} C_{3} C_{4}+s\left\{C_{3}\left(G_{1}+G_{2}\right)+C_{4} G_{2}\right\}+G_{1} G_{2}}{s C_{4}\left\{s\left(G_{1}+G_{2}\right) C_{3}+G_{2} G_{1}\right\}} \\
& =\frac{1}{s C_{4}}+\frac{s C_{4}\left(s C_{3}+G_{2}\right)}{s C_{4}\left\{s\left(G_{1}+G_{2}\right) C_{3}+G_{2} G_{1}\right\}}=\frac{1}{s C_{4}}+\frac{s C_{4}\left(s C_{3}+G_{2}\right)}{s C_{4}\left\{\left(s C_{3}+G_{2}\right) G_{1}+s C_{3} G_{2}\right\}} \\
& =\frac{1}{s C_{4}}+\frac{1}{G_{1}+\left\{\frac{s C_{3} G_{2}}{s C_{3}+G_{2}}\right\}}
\end{aligned}
$$

Figure 10 shows the circuit representation of the output impedance as per Eq. (47).

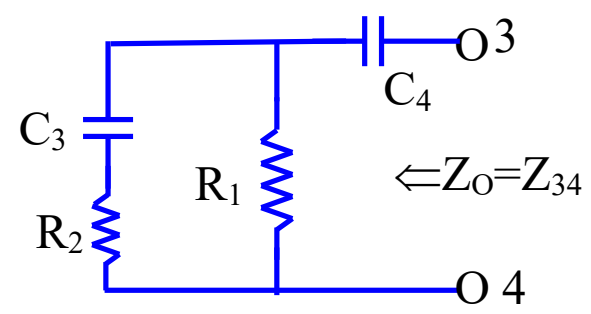

Fig.10 Output impedance representation

\section{RESULTS AND DISCUSSIONS:}

The plots in Figs. 2, 3, 7, and 8 corroborate the theoretical results obtain. The floating admittance mathematical model presented here is so simple that anybody with slight knowledge of electronic devices, but understanding matrix maneuvering can analyse the circuits to derive all types of transfer functions provided the parameters of devices are known to him/ her. The analysis and then designing any circuit using the floating admittance matrix model is based on pure mathematical maneuvering of matrix elements of the circuit. All transfer functions are defined as the ratios of cofactors of first and or second order of the FAM. The mathematical modelling using the FAM approach provides leverage to the designer to comfortably adjust their design style at any analysis stage.

\section{CONCLUSIONS}

A bridged-T network is often used in AC control systems as a filter network. The modelling and simulation of RC bridge -T network has been carried out using MATLAB's Simulink system environment. This paper provides simulated and numerical validated results for both forms of type-1 and type2 RC bridge-T networks used for band pass filter. The MATLAB program developed for the transfer function of both type-1 and type-2 RC bridge-T network have been plotted in the form of magnitude and phase w.r.t. frequency. The input and output impedances are derived and drawn using the FAM technique.

\section{REFERENCES}

[1]. Omar Faruqe, Md. M. M. Saikat, Md. A. K. Bulbul and Md. Tawfiq Amin, "Comparative Analysis and Simulation of Active Inductors for RF Applications in 90 nm CMOS," 3rd International Conference on Electrical Information and Communication Technology (EICT), Khulna, Bangladesh, 7-9 December 2017.

[2]. H.E, Shady Abdel Aleem, Mohamed T. Elmathana and Ahmed F. Zobaa, "Different Design Approaches of Shunt Passive Harmonic Filters Based on IEEE Std. 519-1992 and IEEE Std. 18-2002," Recent Patents on Electrical \& Electronic Engineering 2013, 6, 68-75.

[3]. H.E, Shady Abdel Aleem, Murat Erhan Balci, Selcuk Sakar, "Utilization of cables and transformers using passive filters for non-linear loads," Electrical Power and Energy Systems, ELSEVIER, 71, pp.344-350, 2015.

[4]. M. Bogdan, M. Panu, "Lab View Modeling and simulation of the Low pass and High Pass analog filters," 13th International Conference on Engineering of Modern Electric Systems (EMES), 2015.

[5]. D. S. Sargar, C. L. Bhattar, "LCLR filter design and modelling for harmonic mitigation in interconnected micro grid system," IJRET: International Journal of Research in Engineering and Technology eISSN: 23191163, pISSN: 2321-7308, pp.706-714, Volume: 04 Issue: 04, April-2015

[6]. Selcuk Sakar, Aslan Deniz Karaoglan, Murat Erhan Balci, Shady H. E. Abdel Aleem, Ahmed F. Zobaa, "Optimal Design of Single-Tuned Passive Filters Using Response Surface Methodology," IEEE International School on Nonsinusoidal Currents and Compensation (ISNCC), Łagów, Poland, June 2015 
[7]. Haigh, David G., and Paul M. Radmore, "Admittance matrix models for the nullor using limit variables and their application to circuit design", IEEE Transactions on Circuits and Systems I: Regular Papers vol. 53, No. 10, pp. 2214-2223, October 2006.

[8]. Vago, and E. Hollos, "Two-port models with nullators and norators", Periodica Polytechnica Electrical Engineering 17, no. 4, pp. 301-309, 1973.

[9]. Pragati Kumar, and Raj Senani, "Improved groundedcapacitor SRCO using only a single PFTFN", Analog Integrated Circuits and Signal Processing 50, no. 2, pp. 147-149, 2007.

[10]. Wai-Kai Chen, "Circuit analysis and feedback amplifier theory," CRC Press, 200.

[11]. P. Vizmuller, "RF Design Guide: Systems, Circuits, and Equations," Artech House, ISBN 0-89006-754-6, 1995.

[12]. W. L Everitt, and GE Anner, "Communication Engineering", McGraw-Hill, 1956.

[13]. S.Darlington, "A history of network synthesis and filter theory for circuits composed of resistors, inductors, and capacitors," IEEE Trans. Circuits and Systems, vol 31, pp.3-13, 1984.

[14]. D. Jurišić, G. S. Moschytz, N. Mijat, "low-sensitivity sab band-pass active- $R C$ Filter using impedance tapering," International Symposium on Circuits and Systems (ISCAS 2001), Sydney, pp.I-160-163, Australia, 6-9 May 2001, 0-7803-6685-9/01/C2001 IEEE.

[15]. D.B.S.J. Prasada Rao and B. P. Singh, "A Unified Approach to Electronic Circuit Analysis using the Indefinite Admittance Matrix," International Journal of Electrical Engineering Education, Vol. 15, pp. 267-275, July 1978

[16]. M. Singh, S. K. Roy, and B. P. Singh, "On Demand Realization of Input and Output Resistances of MOSFET Amplifier," American Institute of Physics Conference Proceedings, vol. 1414, no. 1, pp. 266-270, 2011.

[17]. B. P, Singh, and DBSJ Prasada Rao, "A Null Method for Measuring the Parameters of a FET", Int. J. Electronics, Vol.44, pp.193-198, ISSN: 0020-7217, Feb. 1978.

[18]. S. K. Roy, M. Singh, K. K. Sharma, B. Cherry, and B. P. Singh, "Mathematical Modelling of Semiconductor Devices and circuits: A Review;" $3^{\text {rd }}$ International Conference on Intelligent Circuits and Systems (ICICS), June 26-27, 2020,

[19].S. K. Roy, M. Singh, K. K. Sharma, B. Cherry, and B. P. Singh, "Mathematical Modelling of Simple Passive RC Filters using Floating Admittance Technique," IEEE International Conference for Innovation in Technology (INOCON-20), Bengaluru, India. Nov 6-8, 2020.

\section{Creative Commons Attribution License 4.0 (Attribution 4.0 International, CC BY} 4.0)

This article is published under the terms of the Creative Commons Attribution License 4.0 https://creativecommons.org/licenses/by/4.0/deed.en $\underline{\mathrm{US}}$ 